\title{
Functional Roles of a Putative B' Delta Regulatory Subunit and a Catalytic Subunit of Protein Phosphatase 2A in the Cereal Pathogen Fusarium graminearum
}

\author{
Hee-Kyoung Kim and Sung-Hwan Yun* \\ Department of Medical Biotechnology, Soonchunhyang University, Asan 336-745, Korea \\ (Received on May 12, 2012; Revised on May 23, 2012; Accepted on May 23, 2012)
}

Protein phosphatase 2A (PP2A), a family of serine/ threonine protein phosphatases, plays an important role in balancing the phosphorylation status of cellular proteins for regulating diverse biological functions in eukaryotic organisms. Despite intensive studies in mammals, limited information on its role is available in filamentous fungi. Here, we investigated the functional roles of genes for a putative $B^{\prime}$ delta regulatory subunit $(F g P P 2 A R)$ and a catalytic subunit $(F g P P 2 A C)$ of PP2A in a filamentous ascomycete, Fusarium graminearum. Molecular characterization of an insertional mutant of this plant pathogenic fungus allowed us to identify the roles of FgPP2AR. Targeted gene replacement and complementation analyses demonstrated that the deletion of $F g P P 2 A R$, which was constitutively expressed in all growth stages, caused drastic changes in hyphal growth, conidia morphology/germination, gene expression for mycotoxin production, sexual development and pathogenicity. In particular, overproduction of aberrant cylindrical-shaped conidia is suggestive of arthroconidial induction in the $\triangle F g P P 2 A R$ strain, which has never been described in $F$. graminearum. In contrast, the $\triangle F g P P 2 A C$ strain was not significantly different from its wild-type progenitor in conidiation, trichothecene gene expression, and pathogenicity; however, it showed reduced hyphal growth and no perithecial formation. The double-deletion $\triangle F g P P 2 A R ; \triangle F g P P 2 A C$ strain had more severe defects than single-deletion strains in all examined phenotypes. Taken together, our results indicate that both the putative regulatory and catalytic subunits of PP2A are involved in various cellular processes for fungal development in $F$. graminearum.

Keywords : aberrant conidia morphology, B regulatory subunit, catalytic subunit, Fusarium graminearum, protein phosphatase $2 \mathrm{~A}$

\footnotetext{
*Corresponding author.

Phone) +82-41-530-1288, FAX) +82-41-530-3085

E-mail)sy14@sch.ac.kr
}

Molecular regulation of many biological functions in eukaryotic organisms is mediated by protein posttranslational modifications. Protein phosphorylation is one of the major mechanisms in this regulatory system. In particular, reversible phosphorylation of cellular proteins in response to external or intracellular stimuli is an essential step for signal transmission to specific transcription factors that regulate downstream genes in a specific signal transduction cascade (Liu et al., 2000; Luan, 2003). The phosphorylation status of target proteins is modulated by the reversible activities of protein kinases and protein phosphatases. Serine/threonine protein phosphatases (PPs), which catalyze the dephosphorylation of proteins, comprise two different structural families based on biochemical characteristics: type 1 (PP1) and type 2 (PP2). PP2s are subsequently divided into three groups $(2 \mathrm{~A}, 2 \mathrm{~B}$, and $2 \mathrm{C})$. Protein phosphatase $2 \mathrm{~A}(\mathrm{PP} 2 \mathrm{~A})$ holoenzyme, a member of the PP2 family, consists of a core dimer comprising a catalytic $\mathrm{C}$ subunit (PP2AC) and a scaffolding A subunit, which can be complexed with a third variable regulatory $\mathrm{B}$ subunit (PP2AB) to form a heterotrimer (Mumby, 2007). The variable B subunits, which can determine the substrate specificity, catalytic activity, and cellular localization of PP2A, are grouped into four unrelated families, namely $\mathrm{B}$ (PR55), B' (B56/PR61), B" (PR48/59/72/130) and B"' (PR93/110) based on structural similarities. Among the B regulatory subunits, the B' subunit is the most diverse, consisting of five isoforms (alpha, beta, gamma, delta, and epsilon) (McCright et al., 1996; Muneer et al., 2002; Sontag, 2001). The diversity of possible combinations of these subunits allows the PP2A holoenzyme to play roles in the regulation of a wide range of cellular and metabolic processes in a variety of organisms (Gallego and Virshup, 2005; Lechward et al., 2001). Despite intensive investigations in human and model organisms, limited information on the role of PP2A is available in filamentous fungi.

Fusarium graminearum (teleomorph: Gibberella zeae) is a filamentous ascomycetous fungus that threatens both plant and animal health not only by causing disease but also 
by producing mycotoxins in cereal plants (Desjardins, 2006; McMullen et al., 1997). To explore the mechanisms underlying the development of disease caused by this fungus, we have generated a collection of insertional mutants from a representative strain of $F$. graminearum using the restriction enzyme-mediated integration (REMI) procedure (Han et al., 2007; Seo et al., 2007). A screening of REMI transformants resulted in the identification of a mutant that carried a mutation in the gene encoding a putative Bregulatory subunit (B' delta isoform) of PP2A, whose function had not been elucidated in filamentous fungi. Because many fungal traits involved in disease development such as pathogenicity, sexual development, and mycotoxin production are known to be controlled by signal transduction cascades (Yu et al., 2008), this mutant is useful for dissecting the regulatory pathways mediated by PP2A for these traits in F. graminearum. Therefore, the objective of this study was to determine the functional roles of a putative PP2A holoenzyme comprising the variable B regulatory subunit and a putative catalytic subunit in $F$. graminearum using molecular genetic approaches.

\section{Materials and Methods}

Fungal strains and cultures. The wild-type (WT) $F$. graminearum strain used in this study, Z03643, is selffertile and belongs to lineage 7 of the $F$. graminearum species complex (Kim et al., 2011; O'Donnell et al., 2000). The Z43R2629 strain was generated through REMI mutagenesis of Z03643 using the pIGPAPA DNA digested with $K p n I$ (Seo et al., 2007). Fungal strains from $25 \%$ glycerol stock cultures stored at $-80^{\circ} \mathrm{C}$ were maintained on potato dextrose agar (PDA; Difco Laboratories, Detroit, MI, USA). For vegetative growth and conidiation, the strains

Table 1. Primers used in this study

\begin{tabular}{|c|c|c|c|}
\hline Position $^{\mathrm{a}}$ & Name & Nucleotide sequence $^{\mathrm{b}}\left(5^{\prime}-3^{\prime}\right)$ & Amplified region \\
\hline a & phos 5 for & GAGCGCATGTTTTCTAAGG & \multirow{3}{*}{ 5' flank of $F g P P 2 A R$} \\
\hline $\mathrm{b}$ & phos5revtail & GCACAGGTACACTTGTTTAGAGTCCAAAATCGAAGCATCAA & \\
\hline $\mathrm{c}$ & phos5nest & GATGAAGGAGAGGAAAAGAAATGGTGGTAA & \\
\hline $\mathrm{d}$ & phos3fortail & СCTTCAATATCATCTTCTGTCGGTGTCGTGCGGAGGGGCGGTTTA & \multirow{3}{*}{ 3' flank of $F g P P 2 A R$} \\
\hline $\mathrm{e}$ & phos3rev & TACTTCATGGCCTGTGCTTTAGA & \\
\hline $\mathrm{f}$ & phos3nest & TGTGAAGCATTGCGTCGAGTAAAGGTG & \\
\hline $\mathrm{g}$ & Ppe1-5for & GCCATCCTTCACGTTCTTTGCCTTTTTC & \multirow{3}{*}{ 5' flank of $F g P P 2 A C$} \\
\hline $\mathrm{h}$ & Ppe1-5revtail & ACCTCCACTAGCTCCAGCCAAGGATCAAGCATGCCCAGTCG & \\
\hline $\mathrm{i}$ & Ppe1-5nest & CTTCGACTGTTTCCTTTGGTGGTGTTGG & \\
\hline $\mathrm{j}$ & Ppe1-3fortail & TATGAAAATTCCGTCACCAGCCTGTAGAATAGCATAGGGTCATA & \multirow{3}{*}{ 3' flank of $F g P P 2 A C$} \\
\hline $\mathrm{k}$ & Ppe1-3rev & TTTGTACTGAAGCTTAATGTTGAG & \\
\hline 1 & Ppe1-3nest & TCGTGGGAAGAAAGCCGCAGTTGATG & \\
\hline $\mathrm{m}$ & PHO-tailgfp & 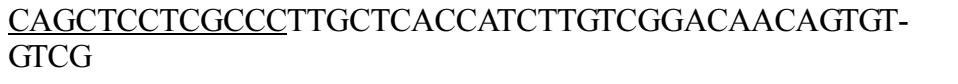 & $\begin{array}{l}\text { a promoter region of } \\
F g P P 2 A R\end{array}$ \\
\hline $\mathrm{n}$ & $\mathrm{GFP} / \mathrm{p} 5$ & ATGGTGAGCAAGGGCGAGGAGCTGT & GFP \\
\hline o & GFP/P6 & CTTGTACAGCTCGTCCATGCCGTGAG & GFP \\
\hline $\mathrm{p}$ & HygB-5 for & CTTGGCTGGAGCTAGTGGAGGT & $h y g B$ \\
\hline q & HygB-3rev & GGCTGGTGACGGAATTTTCATA & hygB \\
\hline $\mathrm{r}$ & Gen/for & CTCTAAACAAGTGTACCTGTGC & gen \\
\hline \multirow[t]{5}{*}{$\mathrm{s}$} & Gen/rev & CGACAGAAGATGATATTGAAGG & gen \\
\hline & 3536 for & AGCCAGCTTATCGCCCTTCC & \multirow{2}{*}{ for qRT-PCR of TRI6 } \\
\hline & 3536rev & AGCCTTTGGTGCCGACTTCTTG & \\
\hline & 8081 for & GTGCATGTTCTGGCCGACCTTGTT & \multirow{2}{*}{$\begin{array}{l}\text { for qRT-PCR of } \\
\text { FGSG_08081.3 }\end{array}$} \\
\hline & 8081 rev & GTGCATGTTCTGGCCGACCTTGTT & \\
\hline
\end{tabular}

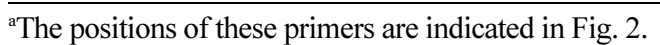

${ }^{b}$ The underlined sequences are complementary to the genes for selectable markers (hygB, gen, or GFP). 
were grown in either PDA, complete medium (CM; Leslie and Summerell, 2006) or YMA medium (Semighini et al., 2008). To induce sexual development, fungal strains were grown on carrot agar plates as described previously (Kim et al., 2008; Leslie and Summerell, 2006). For total RNA extraction under trichothecene-inducing conditions, a fungal strain was inoculated into minimal medium amended with agmatine at a final concentration of $1 \times 10^{4}$ conidia $/ \mathrm{mL}$ and incubated as previously described (Gardiner et al., 2009).

Nucleic acid manipulations and PCR. General procedures for extraction of fungal genomic DNA or total RNA, restriction endonuclease digestion, agarose gel electrophoresis, gel blotting, ${ }^{32} \mathrm{P}$ probe labeling, and hybridization were performed as described previously (Kim et al., 2008; Sambrook and Russell, 2001). The polymerase chain reaction (PCR) primers (Table 1) used in this study were synthesized by the Bioneer oligonucleotide synthesis facility (Bioneer Corp., Chungwon, Korea). Quantitative real-time PCR was performed as previously described (Kim and Yun, 2011) using total RNAs isolated from mycelia grown in the agmatine-amended liquid medium at two time points. Gene expression was measured in two independent samples from each time point using three technical replicates. Gene expression of EF1A (FGSG_08811.3) was used as endogenous controls for data normalization. The amount of each gene transcript from a 4-day-old sample was used as a reference for comparison.

Vector construction. A fungal transforming DNA construct for targeted gene deletion was created using the double-joint PCR method, as described previously (Lee et al., 2009; Yu et al., 2004). DNA fragments corresponding to the 5' and 3' flanking regions of the $F g P P 2 A R$ ORF were amplified from the genomic DNA of Z03643 using the $a / b$ and d/e primer pairs (Table 1 and Fig. 3 for the position of primers), respectively, and mixed with the gen gene cassette amplified with primers $r$ and $s$ (Table 1) from the vector pII99 (Namiki et al., 2001). A second round of PCR using the nested $\mathrm{c}$ and $\mathrm{f}$ primer pair (Table 1) resulted in a $4.1-\mathrm{kb}$ fragment of the final fusion PCR product. For $\triangle F g P P 2 A C$, the $5^{\prime}$ and $3^{\prime}$ flanking regions of the $F g P P 2 A$ ORF were amplified using the $\mathrm{g} / \mathrm{h}$ and $\mathrm{j} / \mathrm{k}$ primer pairs (Table 1 ), respectively, and mixed together with the $h y g B$ gene cassette from the pBCATPH vector (Yun, 1998). The second round of PCR using the nested $\mathrm{i}$ and 1 primer pair (Table 1) generated the final DNA construct for gene deletion. For double deletion of $F g P P 2 A R$ and $F g P P 2 A C$, the $\triangle F g P P 2 A C$ strain was transformed with the DNA construct for $\triangle F g P P 2 A R$ as described above. For molecular complementation, an intact copy of $F g P P 2 A R$ or $F g P P 2 A C$ gene fragments (including its native promoter and terminator regions) was amplified from $\mathrm{Z} 03643$ using the $\mathrm{c} / \mathrm{f}$ and $\mathrm{i} / \mathrm{l}$ primer pairs, respectively, cloned into a pGEM-T Easy vector (Promega), and added to the $\triangle F g P P 2 A R$ protoplasts along with the pBCATPH vector carrying $h y g B$, or to the $\triangle F g P P 2 A C$ protoplasts along with pII99 carrying gen.

Fungal transformation and virulence test. All PEGmediated fungal transformation steps using the DNA constructs described above were performed as described previously (Kim et al., 2008; Kim et al., 2011). The virulence of the fungal strains was determined on young ears of corn or on spikelets of barley as described previously (Han et al., 2007; Lee et al., 2009).

\section{Results}

Phenotypes and molecular characterization of the REMI mutant Z43R2629. A REMI mutant of $F$. graminearum, designated Z43R2629, showed pleiotropic phenotypes in several traits such as hyphal growth, pigmentation, sexual development, and pathogenicity on corn ears (Fig. 1). Compared to its WT progenitor Z03643, the radial hyphal growth of Z43R2629 was reduced to $\sim 30 \%$ of that of Z03643, with fewer aerial mycelia and no red pigmentation when grown on PDA or carrot agar (Fig. 1). Under the sexual induction condition, Z43R2629 formed no perithecia even 6 weeks after removing the aerial mycelia that had already grown on carrot agar for 1 week; the WT strain usually began to form fertile perithecia 4 days after sexual induction (Fig. 1). In addition, the ability of Z43R2629 to cause disease symptoms was almost abolished on young corn ears, whereas inoculation with Z03643 resulted in a large area of necrosis around the inoculation points (Fig. 1).

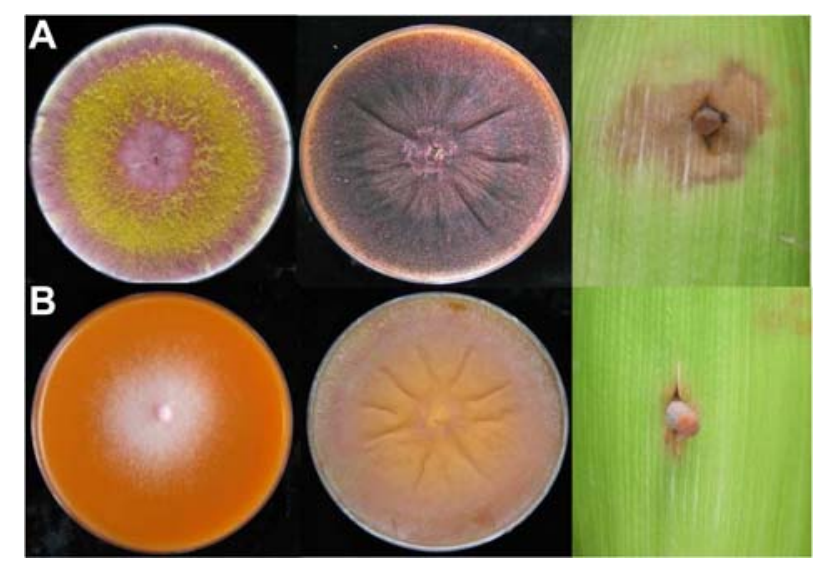

Fig. 1. Comparison of phenotypes in the Fusarium graminearum WT Z03643 strain (A) and Z43R2629 strain (B). Growth of aerial mycelia on carrot agar, perithecia formation on carrot agar, and symptoms on corn ears are shown in the left, center, and right panels, respectively. 
A

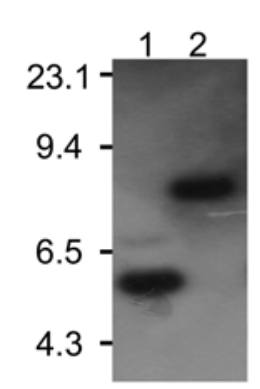

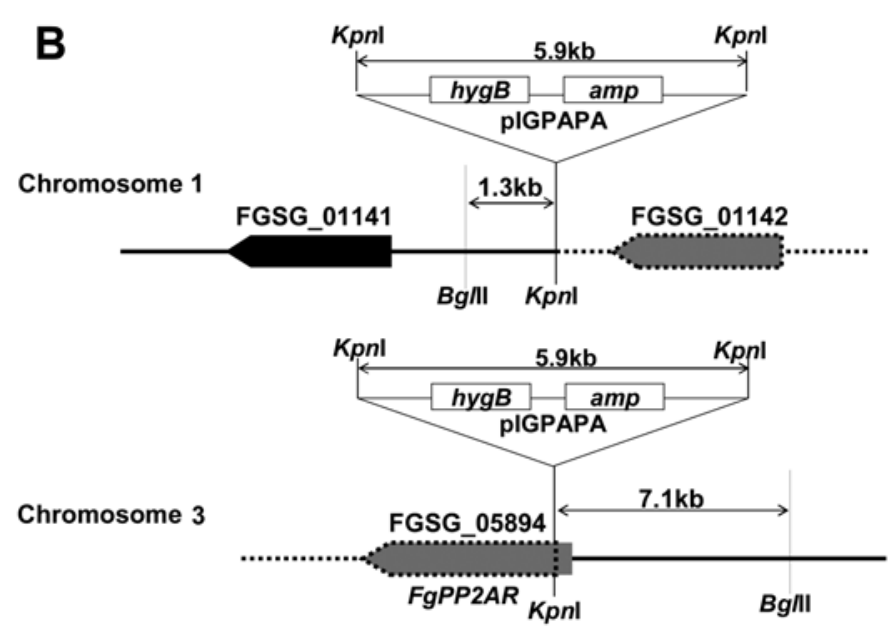

Fig. 2. Molecular analyses of the vector insertions in the Z43R2629 genome. (A) A blot of genomic DNA of Z43R2629 digested with $K p n I$ (lane 1) and BglII (lane 2) was hybridized with pIGPAPA. The sizes of lambda DNA standards (in kilobases) are indicated to the left of the blot. (B) Molecular structures of the vector insertion site in Z43R2629. The open reading frames (ORFs) near the vector insertion point are indicated by the filled arrow. The dashed lines represent the genomic regions that are not present at the vector insertion points in Z43R2629, probably due to a rearrangement between chromosomes 1 and 3.

The vector insertion point in the Z43R2629 genome was identified using DNA gel blot analysis (Fig. 2). In genomic DNA digested with KpnI, the enzyme used to linearize the vector DNA (pIGPAPA) in the REMI procedure, the entire sequence of pIGPAPA hybridized to a single $\sim 5.9-\mathrm{kb}$ fragment the same size as pIGPAPA (Fig. 2A), indicating a single vector insertion in the Z43R2629 genome, typical of a real REMI event. When the genomic DNA was digested with $B g / I I$, for which the vector has no recognition site, a single hybridizing band of $\sim 8.4 \mathrm{~kb}$ appeared (Fig. 2A). Nucleotide sequencing of the 8.4-kb BgIII fragment recovered using a plasmid rescue procedure revealed that it contains $1.3 \mathrm{~kb}$ of genomic DNA to the left side and $7.1 \mathrm{~kb}$ to the right side of the vector. However, BLAST searches of the F. graminearum genome database (http:// www.broadinstitute.org/annotation/genome/fusarium_group/ MultiHome.html) revealed that the immediate left and right flanking regions could not be connected into a continuous open reading frame $(\mathrm{ORF})$. Instead, the left flanking sequence of the vector started at a KpnI site $230 \mathrm{bp}$ downstream of the putative stop codon of an ORF (annotated as FGSG_01142 in the fungal genome) located on chromosome 1 , while the right flanking sequence started at a $K p n I$ site within an ORF (FGSG_05894) of 655 amino acids (interrupted by four putative introns) located on chromosome 3 (Fig. 2B). The entire deduced sequence of FGSG_05894, designated $F g P P 2 A R$, had high similarity to the putative $\mathrm{B}^{\prime}$ (also known as B56)-delta regulatory subunit of protein phosphatase 2A. This vector insertion pattern suggests that chromosomal rearrangement occurred during REMI mutagenesis in the genome of Z43R2629.
Targeted deletions of FgPP2AR and FgPP2AC. To confirm that the disruption of $F g P P 2 A R$ was a direct cause of pleiotropic changes in Z43R2629, the genomic copy of $F g P P 2 A R$ was deleted from the genome of $F$. graminearum Z03643 strain using a targeted gene replacement strategy (Fig. 3). Double crossover integration of the fusion PCR product carrying a gen cassette fused to the $5^{\prime}(1.0 \mathrm{~kb})$ and $3^{\prime}(1.0 \mathrm{~kb})$ regions flanking $F g P P 2 A R$ into the fungal genome resulted in the deletion of $F g P P 2 A R$ (designated $\triangle F g P P 2 A R$ ) (Fig. 3A). Targeted gene deletion was verified by DNA gel blot analysis (Fig. 3A). When genomic DNA from the $\triangle F g P P 2 A R$ strains was digested with $N c o$ I, a single 4.7-kb hybridizing band was observed, instead of the 6.9-kb band that hybridized in Z03643, confirming that the entire $F g P P 2 A R$ ORF had been deleted and replaced with $g e n$, as expected in the $\triangle F g P P 2 A R$ strains. All of the examined $\triangle F g P P 2 A R$ strains exhibited the same pleiotropic changes on PDA and carrot agar as did the REMI mutant Z43R2629 (Figs. 1 and 4).

To determine the function of a putative catalytic subunit of the protein phosphatase 2A (PP2A) in F. graminearum, the $F g P P 2 A C$ gene was deleted from the $\mathrm{Z} 03643$ genome, as described above. $F g P P 2 A C$, annotated as FGSG_01464.3 in the genome database, is an ortholog of the F. verticillioides CPP1 encoding a putative PP2A catalytic subunit (Choi and Shim, 2008). The entire 401-amino acid ORF of $F g P P 2 A C$ was replaced with a $h y g B$ cassette by double cross-over between a fusion PCR construct and the fungal genome, which was verified by DNA gel blot hybridization (Fig. 3B). The BglII-digested genomic DNA from the $\triangle F g P P 2 A C$ strains showed a single $17.0-\mathrm{kb}$ hybridizing 
A

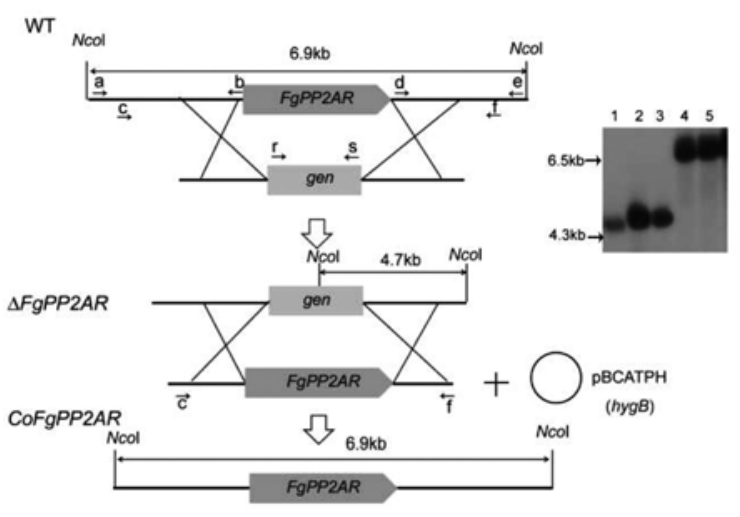

C

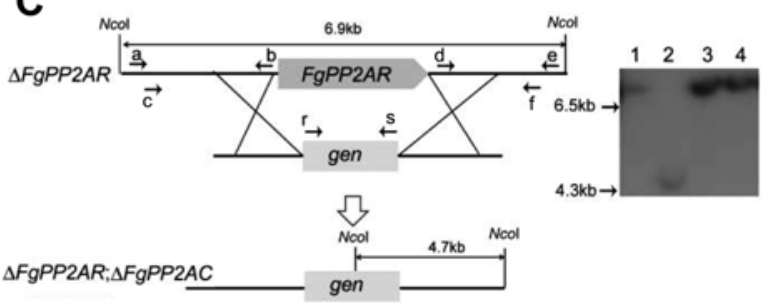

B

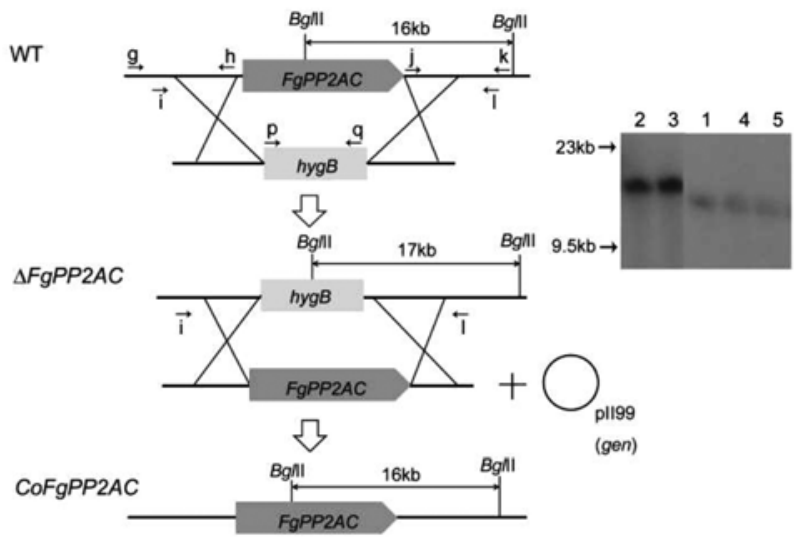

Fig. 3. Targeted deletions of $F g P P 2 A R(\mathrm{~A}), F g P P 2 A C(\mathrm{~B})$, and both genes (C) from the genome of $F$. graminearum WT strain Z03643, and genetic complementation. (Left in each panel) Deletion and complementation strategies. WT: genomic DNA of Z03643, $\triangle F g P P 2 A R$ : the Z03643 strain with $F g P P 2 A R$ deleted, $\triangle F g P P 2 A C$ : the strain with $F g P P 2 A C$ deleted, $\triangle F g P P 2 A R ; \triangle F g P P 2 A C$ : the strains carrying the double gene deletion, $C o F g P P 2 A R$ : the $\triangle F g P P 2 A R$ carrying an intact copy of $F g P P 2 A R$ ORF, and $C o F g P P 2 A C$ : the $\triangle F g P P 2 A C$ carrying an intact copy of $F g P P 2 A C$ ORF. The transcription direction of each gene is indicated by the filled arrow. The positions of primers used in the construction of transforming vectors (see the Materials and Methods section) are indicated by arrows with the primer names (see Table 1). (Right in each panel) Gel blots of NcoI-digested genomic DNA (A and C) and BglII-digested genomic DNA (B) from transgenic strains, probed with the DNA fragments amplified with primer pairs $\mathrm{d} / \mathrm{f}$ and $\mathrm{j} / \mathrm{k}$, respectively. Lanes in A and B: 1, Z03643; 2-3, the gene deletion strains; 4-5, the complemented strains. Lanes in C: 1, the $\triangle F g P P 2 A C$ strain; 2 , the double gene deletion strain.

band, instead of the 16.0-kb band in Z03643 (Fig. 3B). To construct the transgenic $F$. graminearum strain lacking both $F g P P 2 A R$ and $F g P P 2 A C(\triangle F g P P 2 A R ; \triangle F g P P 2 A C)$, the genomic copy of $F g P P 2 A R$ was deleted from the genome of a $\triangle F g P P 2 A C$ strain using the same strategy as described for the deletion of $F g P P 2 A R$ from Z03643 (Fig. 3C); the double gene deletion was confirmed by DNA gel blot analysis (Fig. 3C).

Genetic complementation of $\triangle F g P P 2 A R$ and $\triangle F g P P 2 A C$. For further confirmation of the gene function in $F$. graminearum, an intact copy of $F g P P 2 A R$ or $F g P P 2 A C$ was introduced into the genome of a $\triangle F g P P 2 A R$ or a $\triangle F g P P 2 A C$ strain, respectively. A 4.0-kb FgPP2AR DNA fragment including $5^{\prime}(1.0 \mathrm{~kb})$ and $3^{\prime}(1.0 \mathrm{~kb})$ flanking sequences was amplified from the genomic DNA of Z03643 using the $\mathrm{c}$ and $\mathrm{f}$ primer pair (Table 1) and directly added into protoplasts of the $\triangle F g P P 2 A R$ strain, along with pBCATPH carrying hygB as a fungal selectable marker
(Fig. 3A). A total of 8 of 18 hygB $^{\mathrm{R}}$ transformants were no longer resistant to geneticin, suggesting the $\mathrm{FgPP} 2 \mathrm{AR}$ gene was integrated into its original genomic locus of the $\triangle F g P P 2 A R$ strain by double cross-over, as confirmed by DNA gel blot analysis (Fig. 3A). Similarly, the deletion of $F g P P 2 A C$ was successfully complemented with $F g P P 2 A C$ using a co-transformation procedure with pII 99 carrying gen (Fig. 3B).

Aberrant morphology caused by the gene deletions. The hyphal growth pattern of the $\triangle F g P P 2 A R$ strain was similar to that of the REMI mutant Z43R2629. Compared to the WT Z03643 strain, it produced less aerial mycelia with reduced radial growth and no red pigmentation on carrot agar (Figs. 1 and 4). The $\triangle F g P P 2 A C$ strain also showed a reduced radial growth similar to the $\triangle F g P P 2 A R$ strain, but its mycelia were more heavily pigmented with purple/red (Fig. 4). The $\triangle F g P P 2 A R ; \triangle F g P P 2 A C$ strains exhibited less than $10 \%$ of the WT level of radial growth with no 


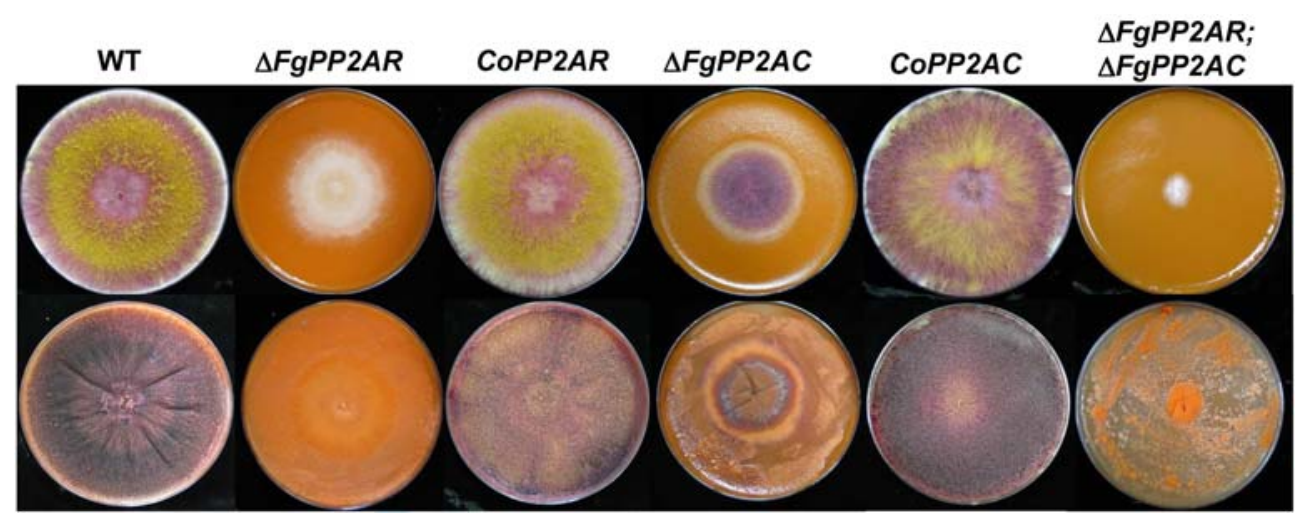

Fig. 4. Mycelial growth (upper panel) and perithecia formation (lower panel) of the transgenic F. graminearum strains generated in this study on carrot agar. The names and descriptions of each strain are provided in the legend of Fig. 3.

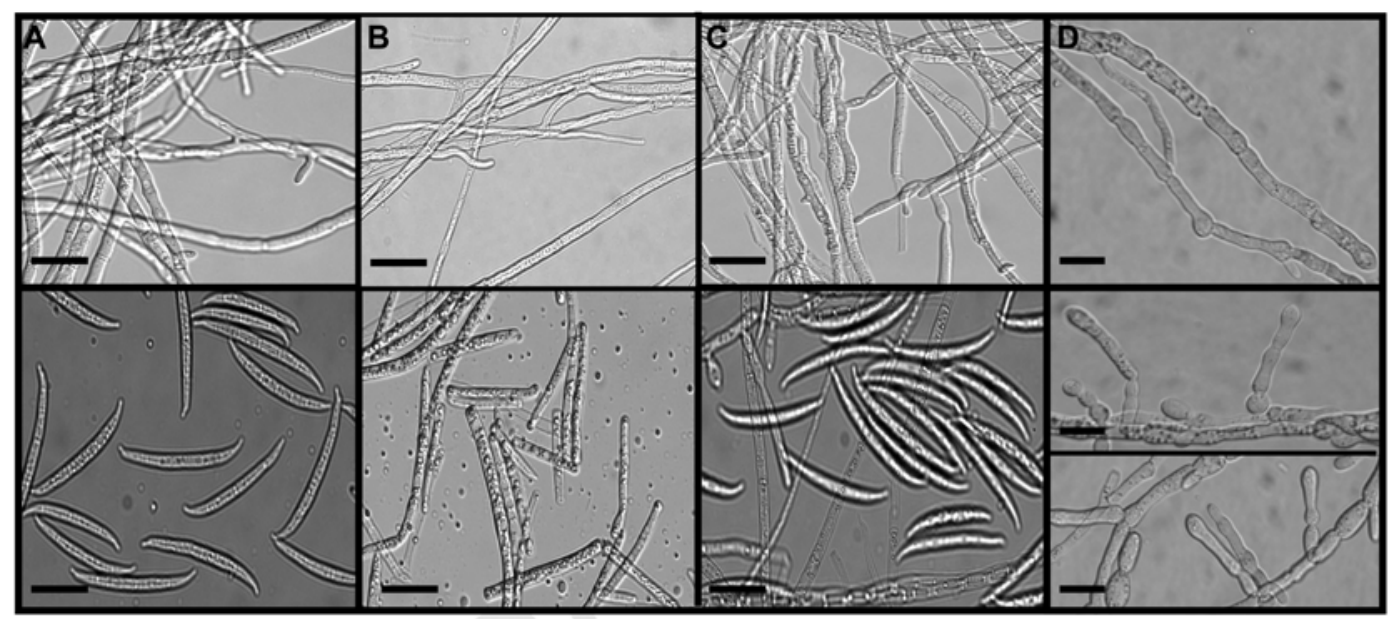

Fig. 5. Hyphal growth (upper panels) and conidia morphology of the gene deletion strains grown on YMA medium. (A) Z03643, (B) $\triangle F g P P 2 A R$ strain, (C) $\triangle F g P P 2 A C$ strain, (D) $\triangle F g P P 2 A R ; \triangle F g P P 2 A C$ strain. Scale bar $=100 \mu \mathrm{m}$.

pigmentation (Fig. 4). In hyphal morphology, both the $\triangle F g P P 2 A R$ and $\triangle F g P P 2 A C$ strains were not significantly different from the WT strain, but the double deletion stain showed thicker and swollen hyphae (Fig. 5).

The deletion of $F g P P 2 A R$ led to an aberrant conidial shape and germination in F. graminearum (Figs. 5 and 6). The 3-5 septate macroconidia formed on aerial mycelia in Z03643 were hyaline, slightly curved, and tapering at both ends with a foot-celled base, typical of the conidia morphology of $F$. graminearum (Fig. A). In contrast, the $\triangle F g P P 2 A R$ strain produced no typical macroconidia, instead forming a long cylindrical-shaped conidia with a rounded apex and a truncate, rounded base with variable sizes (Fig. 5B). The double-deletion strain produced neither the typical falcate nor truncate conidia observed in the $\mathrm{Z} 03643$ and $\triangle F g P P 2 A R$ strains, respectively. Instead, its hyphae became thicker than those from these two strains, and the hyphal tips were swollen from which minor constriction chains were probably made. In addition, it produced hyphal frag- ments with swollen intercalary and terminal cells (or minor constructions; Fig. 5D). However, it is unclear whether these aberrant structures resulted from the ultimate disarticulation of the hyphal chains with minor constrictions. However, the morphology of conidia formed in the $\triangle F g P P 2 A C$ strain was not different from that in $\mathrm{Z} 03643$ (Fig. 5C). Within $3 \mathrm{~h}$ of incubation, the conidia from both the $\mathrm{Z} 03643$ and $\triangle F g P P 2 A C$ strains were swollen and began to form germination tubes from terminal cells, intercalary cells, or both at $5 \mathrm{~h}$ (Fig. 6A and C). However, the truncate conidia of the $\triangle F g P P 2 A R$ strain formed the germ tube at $7-9 \mathrm{~h}$ from terminal regions only (Fig. 6B). In the double-deletion strain, germ tubes started to emerge from swollen cells in both ends of arthroconidia-like structures at $3-5 \mathrm{~h}$, but elongated more slowly compared to the case of Z03643 (Fig. 6D). These aberrant morphologies were restored to the WT level when an intact copy of $F g P P 2 A R$ or $F g P P 2 A C$ was introduced into the $\triangle F g P P 2 A R$ or $\triangle F g P P 2 A C$ strain, respectively (data not shown). 


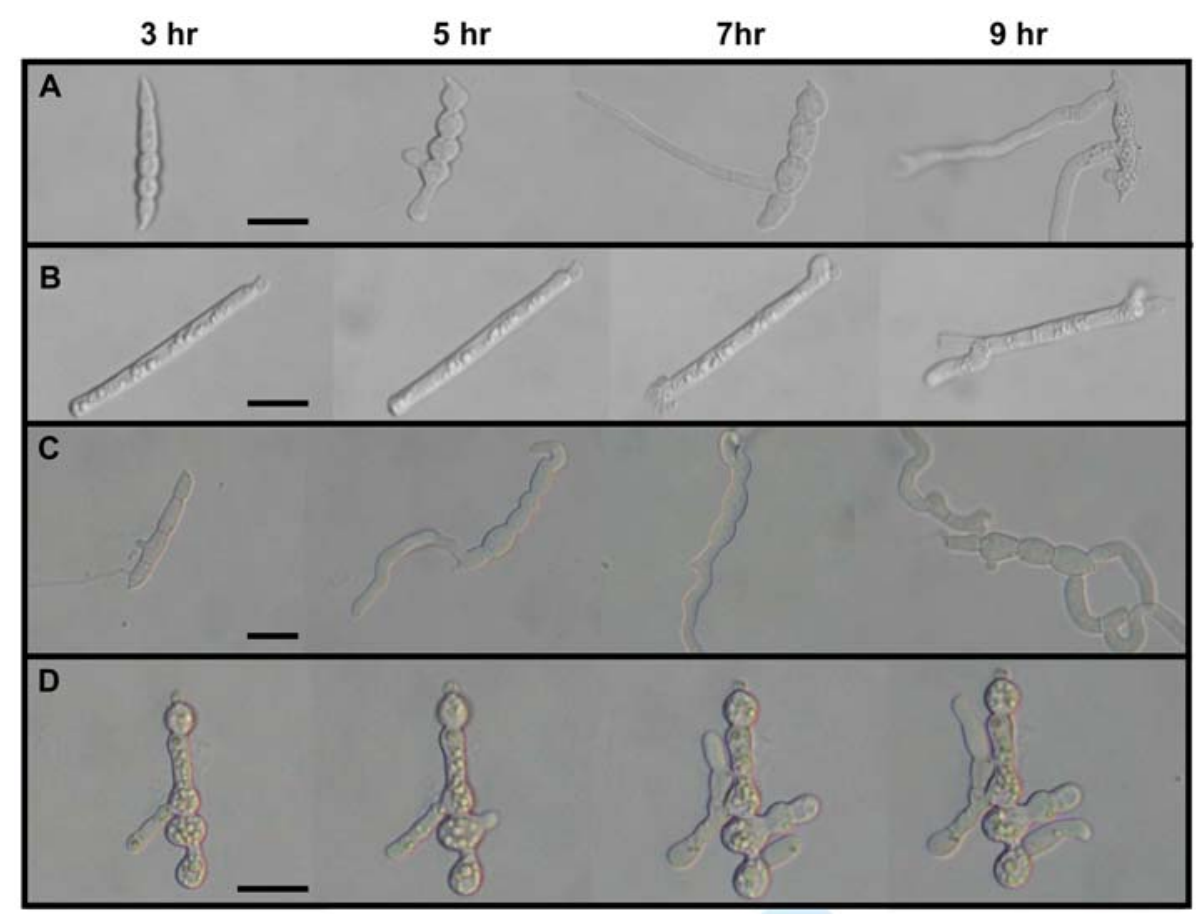

Fig. 6. Conidial germination of the gene deletion strains using complete medium (CM). (A) Z03643, (B) $\triangle F g P P 2 A R$ strain, (C) $\triangle F g P P 2 A C$ strain, (D) $\triangle F g P P 2 A R ; \triangle F g P P 2 A C$ strain. Times elapsed (in h) after inoculation into $\mathrm{CM}$ are indicated above the panels. Scale bar $=100 \mu \mathrm{m}$.

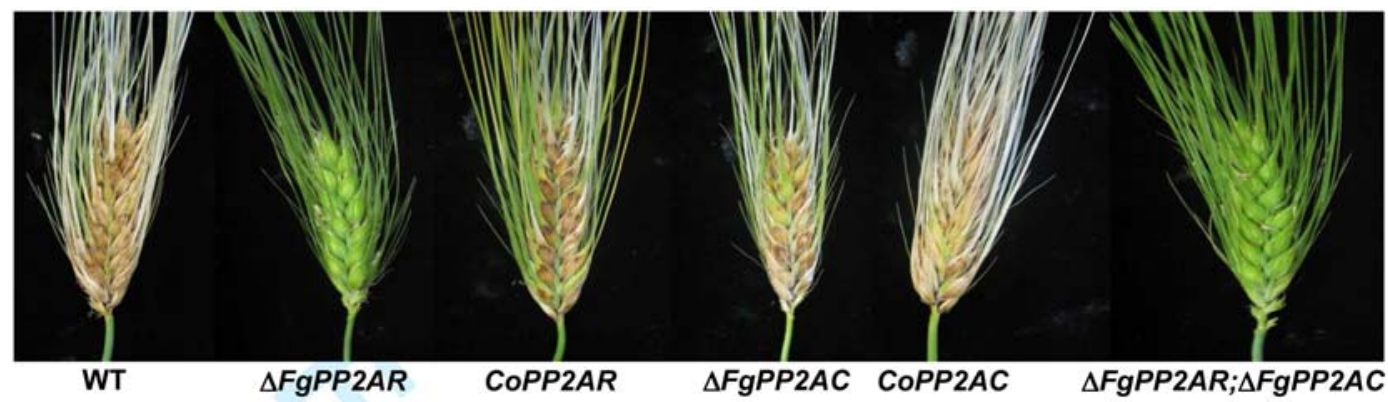

Fig. 7. Pathogenicity of the gene deletion strains on barley heads.

Defects in sexual development and virulence. The capacity of the $\triangle F g P P 2 A R$ strain for sexual reproduction was completely impaired as described above in the REMI mutant Z43R2629 (Fig. 4). In addition, the $\triangle F g P P 2 A C$ and double-deletion strains were unable to form perithecia even after more than 6 weeks; the WT Z03643 strain formed abundant perithecia containing ascospores on carrot agar within 2 weeks (Fig. 4).

All of the sexual defects in these gene deletion strains were restored to the WT level when genetically complemented in strains $\triangle F g P P 2 A R:: F g P P 2 A R$ and $\triangle F g P P 2 A C:$ : $F g P P 2 A C$ (Fig. 4). In addition, we examined the pathogenic development of the gene deletion strains on a host plant. Inoculation with the $\triangle F g P P 2 A R$ strain had almost no effect on barley heads, resulting in no development of disease symptoms (Fig. 7). The abolished virulence was also evident in the double-deletion strain (Fig. 7). Unlike these strains, the $\triangle F g P P 2 A C$ strain caused necrotic spots on approximately $70-80 \%$ of spikelets of each barley head examined by 10 days after inoculation, and these symptoms developed into a typical head blight as severe as those caused by the Z03643 strain (Fig. 7). The WT level symptoms were observed when each genetically complemented strain described above was inoculated (Fig. 7).

Changes in expression of secondary metabolite genes. To determine if $F$. graminearum protein phophatase $2 \mathrm{~A}$ is involved in expression of genes responsible for biosyntheses of several toxic metabolites, we quantified the relative expression level of two genes (TRI6 and FGSG_08081.3), 
TRI6

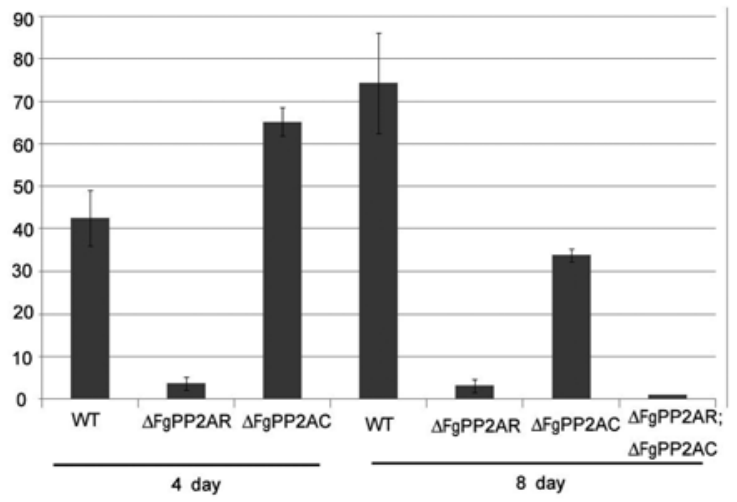

FGSG_08081

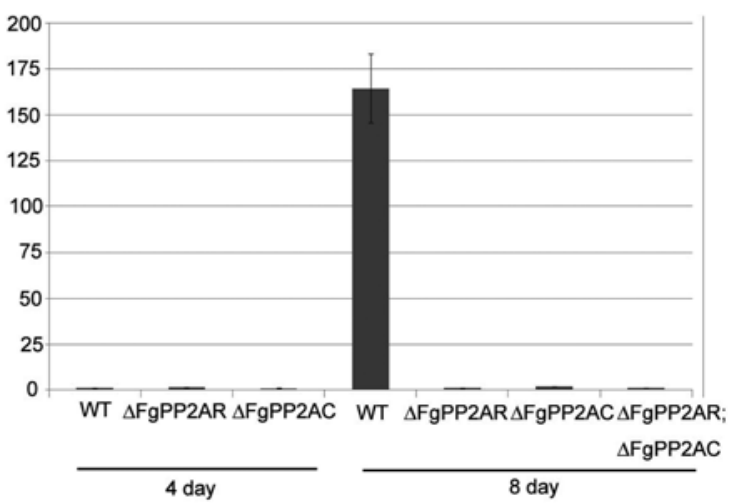

Fig. 8. The expression pattern of TRI6 and FGSG_08081.3 in agmatine-amended liquid medium for the production of trichothecenes and butenolide determined using quantitative real-time PCR. The numbers on the y-axis represent the relative amount of each gene transcript. The numbers on the $\mathrm{x}$-axis indicate the names of deletion strains grown in the liquid medium for 4 and 8 days. Error bars represent standard error.

which were involved in the production of the mycotoxins trichothecene and butenolides in the agmatine-amended medium. The expression level of TRI6 was significantly ( $p$ $<0.05)$ reduced in both the $\triangle F g P P 2 A R$ and the doubledeletion strains, whereas expression in the $\triangle F g P P 2 A C$ strain was not different compared to those in the WT strain when grown in agmatine-amended liquid medium, an inducing condition for the production of trichothecenes and butenolide. The gene expression level from the double deletion mutant at day 4 was not available due to insufficient mycelia growth at that time point (Fig. 8). Unlike the case of TRI6, the expression of FGSG_08081.3 was dramatically down-regulated in all of the deletion strains at day 8 in the agmatine medium (Fig. 8).
A

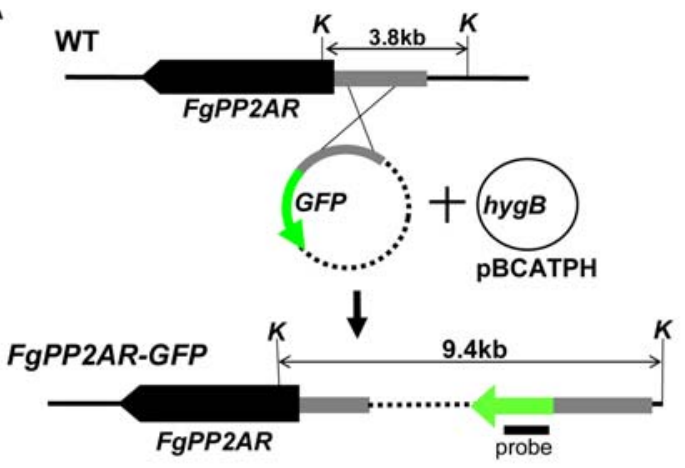

C

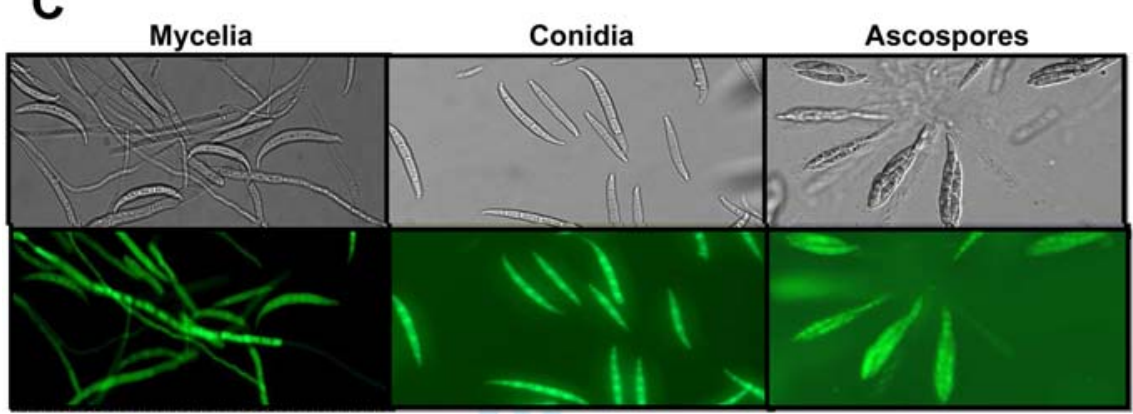

B

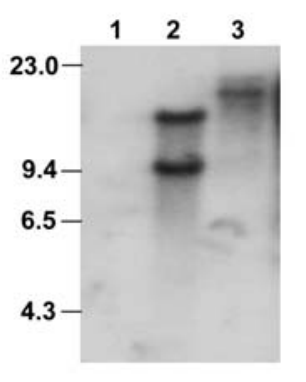

Fig. 9. Expression of GFP under control of a putative promoter region of $F g P P 2 A R$. (A) Scheme for introduction of the GFP gene into the fungal genome. The fusion PCR product of the promoter region and GFP gene, amplified with primer pairs $\mathrm{m} / \mathrm{f}$ and $\mathrm{d} / \mathrm{n}$ (Table 1), was cloned into pGEMT, and added to the Z03643 strain along with pBCATPH. (B) DNA gel blot of hygromycin B-resistant transformants of Z03643 probed with the GFP gene. Lanes 1, Z03643; 2-3, hygB ${ }^{\mathrm{R}}$ transformants. (C) Observation of mycelia, conidia, and ascospores under light (upper panels) and fluorescence (lower panels) microscopes. 
Expression of green fluorescence protein under a putative promoter region of $\boldsymbol{F g P P 2 A R}$. To determine the expression pattern of $F g P P 2 A R$ during the entire growth stages of $F$. graminearum, plasmid DNA carrying the GFP gene under the control of a $1.0 \mathrm{~kb}$ putative promoter region of FgPP2AR was introduced into the Z03643 strain along with pBCATPH (Fig. 9A). All of the hygromycin Bresistant transformants carrying the GFP gene construct, confirmed by DNA gel blot analysis (Fig. 9B), expressed GFP in the stages of mycelia, conidia, and ascospores at all examined time points, confirming that $F g P P 2 A R$ was expressed constitutively in all of the cell types (Fig. 9C).

\section{Discussion}

Despite the chromosomal rearrangement at the vector insertion region in a REMI mutant Z43R2629, the molecular characterization of Z43R2629 allowed the identification of the functional roles of a putative delta isoform of B' (B56) regulatory subunit of $\mathrm{PP} 2 \mathrm{~A}$ (FgPP2AR) in $F$. graminearum, which had never been characterized in filamentous fungi. Although the $\mathrm{B}^{\prime}$ regulatory subunit is the most diverse class among the three families (B, B', and B") of the B-regulatory subunits, its functional roles have been intensively determined only in mammals (Bobulescu et al., 2010; Flegg et al., 2010; Hahn et al., 2010; Louis et al., 2011; Muneer et al., 2002). Specifically expressed in both embryonic cells and brain, it is involved in the dephosphorylation of proteins in various signaling pathways (McCright et al., 1996). The only B-regulatory subunit of PP2A whose functions have been analyzed in filamentous fungi is the B class subunit encoded by $r g b-1$ and its homolog in $N$. crassa and S. sclerotinia, respectively (Erental et al., 2007; Yang et al., 2004; Yatzkan and Yarden, 1999). Among the pleiotropic changes caused by $\triangle F g P P 2 A R$, which were confirmed by gene deletion and complementation analyses, the phenotype of aberrant conidia morphology has never been described in the various $F$. graminearum mutants. The aberrant cylindrical-shaped conidia predominantly found in the $\triangle F g P P 2 A R$ strain, which lacks both the notched apex and classic Fusarium foot-celled base, seem to be similar to arthroconidia, and have never been observed in F. graminearum. This abnormal conidiation in the $F$. graminearum $\triangle F g P P 2 A R$ strain is comparable to that in the $N$. crassa rgb- $1^{R I P}$ strain (Yatzkan and Yarden, 1999). In WT $N$. crassa, cylindrical-shaped arthroconidia comprise roughly $1 \%$ of the total conidial mass (Springer and Yanofsky, 1989), whereas the $r g b-1$ mutant shows overproduction of arthroconidia along with reduced normal macroconidiation (Yatzkan and Yarden, 1999). If the aberrant conidia in the $\triangle F g P P 2 A R$ are true arthroconidia, it is likely that FgPP2AR participates in the regulatory or signaling pathway(s) required for normal conidiation in F. graminearum, which might be common in those controlled by RGB-1 in $N$. crassa. The induced arthroconidiation-like development was more evident when $F g P P 2 A C$ encoding a putative catalytic subunit of PP2A was deleted in the genetic background of $\triangle F g P P 2 A R$. Because the single deletion of $\mathrm{FgPP} 2 \mathrm{AC}$ caused no changes in conidia morphology, formation of frequent constrictions in hyphal chains and arthroconidia-like hyphal fragments in the double-deletion strain could be caused by an additive effect of $\triangle F g P P 2 A R$. In particular, the swollen and spherical structures formed at both ends of the hyphal fragments during germination are similar to those of arthroconidia in N. crassa (Springer and Yanofsky, 1989). However, this functional analogy between FgPP2AR and RGB-1 needs further consideration and experimental confirmation. The B-regulatory subunits encoded by these two genes belong to different classes (B' delta and B classes for F. graminearum and $N$. crassa, respectively). It remains unclear whether the enhanced or induced arthroconidiation would be a common phenotypic change in the fungal strains lacking B-regulatory subunits. In this respect, the fact that no changes in conidia morphology were observed in the rgb-1 mutant of S. sclerotinia could argue against this hypothesis (Erental et al., 2007). It is also necessary to disrupt the $r g b$ - 1 ortholog (FGSG_01369.3) in F. graminearum to determine whether it induces arthroconidiation as in the $\triangle F g P P 2 A R$ strain. Further microscopic analyses are necessary to elucidate the developmental processes involved in the production of the arthroconidia-like structure because hyphal fragmentations into arthroconidia were not clearly observed in the $\triangle F g P P 2 A R$ strain. Another possible scenario for the abnormal conidial morphology may be that conidium initials formed on a phialide were released without undergoing proper maturation for normal macroconidiation in the $\triangle F g P P 2 A R$ strain. Although further investigations are required to explore the mechanism underlying these defects, it is clear that FgPP2AR directs the holoenzyme of FgPP2A to control genes or proteins necessary for proper conidiogenesis in F. graminearum. Despite the uncertainties regarding conidiation, the defects in conidial morphology and germination (altered germination polarity) could be attributed, at least in part, to non-pathogenicity on host plants and self-sterility of the $\triangle F g P P 2 A R$ strain. It is also likely that $\triangle F g P P 2 A R$ may cause inappropriate expression of a variety of genes or proteins involved in sexual development and pathogenicity. Altered pigmentation of aerial mycelia and reduced transcription of genes for trichothecenes and butenolide in the $\triangle F g P P 2 A R$ strain also suggest that secondary metabolism is regulated by pathways controlled by FgPP2AR in F. graminearum. Constitutive GFP expression under the control of the $F g P P 2 A R$ 
promoter in mycelia, conidia, and ascospores indicates that $F g P P 2 A R$ is ubiquitously expressed during all developmental stages (both asexual and sexual reproduction stages), which is comparable to the high expression of the $\mathrm{B}^{\prime}$ delta subunit gene in human brain and embryo (McCright et al., 1996).

In contrast to $F g P P 2 A R$, the deletion of $F g P P 2 A C$ encoding a putative catalytic subunit of PP2A has milder effects on the physiology of $F$. graminearum, although mycelial growth in the $\triangle F g P P 2 A C$ strain was reduced to the level similar to that in the $\triangle F g P P 2 A R$ strain. To date, the functional roles of two catalytic subunits of PP2A have been investigated in filamentous fungi: a homolog of $N$. crassa pph-1 (Erental et al., 2007; Kosmidou et al., 2001; Yatzkan et al., 1998) and that of N. crassa ppe-1 (Choi and Shim, 2008). The former was confirmed to be essential for fungal growth in N. crassa, S. screlotinia, and A. nidulans because complete or partial gene inactivation led to lethality or slow hyphal growth. Due to their essential functions, the specific roles of these homologs could not be determined in fungal phenotypes except for hyphal growth. The latter, to which FgPP2AC in this study is orthologous (with $75 \%$ amino acid identity), is known to be involved in hyphal growth, conidial development/germination, hyphae morphology, and biosynthesis of a polyketide mycotoxin (fumonisin) in $F$. verticillioides, suggesting that this PP2AC is associated with multiple downstream signaling pathways in $F$. verticillioides. However, most phenotypic changes in the $F$. verticillioides strain lacking this ortholog $(C P P 1)$ were different from or not compatible to those in the $F$. graminearum $\triangle F g P P 2 A C$ strain, even though $C P P 1$ displayed high similarity to $F g P P 2 A C$ (with $94 \%$ amino acid identity). For example, hyphal swelling and reduced conidia germination rates in the $F$. verticillioides $\triangle C P P 1$ strain were not observed in the $\triangle F g P P 2 A C$ strain. The specific role of $C P P 1$ in probable negative regulation of the FUM1 gene for fumonisin production in $F$. verticillioides cannot be directly compared to that of $F g P P 2 A C$ because no functional homolog of FUM1 is present in $F$. graminearum. Reduced expression of the gene for butenolide, but not for trichothecene in the $\triangle F g P P 2 A C$ strain, supports the specific but different role(s) of $F g P P 2 A C$ in secondary metabolism compared to $F$. verticillioides. The phenotypes of pathogenicity and sexual development caused by $\triangle F g P P 2 A C$ cannot be compared to those by $\triangle C P P 1$ because the effect of $\triangle C P P 1$ in these traits was not investigated in F. verticillioides (Choi and Shim, 2008). These phenotype comparisons imply that $\mathrm{FgPP} 2 A C$ and its ortho$\log C P P 1$ have overlapping functions in hyphal growth, but may participate in different regulatory pathways for other traits in two closely related fungal species. In addition, the milder effects of $\triangle F g P P 2 A C$ than $\triangle C P P 1$ and even $\triangle F g P P 2 A R$ suggest that the function of FgPP2AC may be redundant in $F$. graminearum. To confirm this, deletion of the gene (FGSG_09815.3) orthologous to the $N$. crassa pph-1 from $F$. graminearum would be necessary. However, more severe changes in all of the phenotypes examined in the double gene deletion $(\triangle F g P P 2 A R ; \triangle F g P P 2 A C)$ strain, some of which were indistinguishable from the WT strain in the $\triangle F g P P 2 A C$ strain, indicates that a FgPP2A holoenzyme probably consisting of FgPP2AC and FgPP2AR is involved in controlling various regulatory and signal transduction pathways required for hyphal growth, conidiation, sexual development, mycotoxin production, and pathogenicity in F. graminearum. To explore the specific role(s) of these PP2A subunits in the physiology of F. graminearum, further investigations are necessary, such as the identification of downstream genes and target proteins for dephosphorylation, and confirmation of functional FgPP2A holoenzyme formation from FgPP2AC and FgPP2AR.

\section{Acknowledgments}

This work was supported by the Agricultural Research Center program of the Ministry for Food, Agriculture, Forestry and Fisheries, Korea, and by grants from "Cooperative Research Program for Agricultural Science \& Technology Development (Project No. PJ007340)" and the Next-Generation BioGreen 21 Program (No. PJ008210), Rural Development Administration, Republic of Korea.

\section{References}

Bobulescu, I. A., Quinones, H., Gisler, S. M., Di Sole, F., Hu, M. C., Shi, M., Zhang, J., Fuster, D. G., Wright, N., Mumby, M. and Moe, O. W. 2010. Acute regulation of renal $\mathrm{Na}+/ \mathrm{H}+$ exchanger NHE3 by dopamine: Role of protein phosphatase 2A. Am. J. Physiol. Renal Physiol. 298:F1205-F1213.

Choi, Y. E. and Shim, W. B. 2008. Functional characterization of Fusarium verticillioides $C P P 1$, a gene encoding a putative protein phosphatase 2A catalytic subunit. Microbiology 154: 326-336.

Desjardins, A. E. 2006. Fusarium mycotoxin: Chemistry, genetics and biology. APS Press, St. Paul, MN, U.S.A.

Erental, A., Harel, A. and Yarden, O. 2007. Type 2A phosphoprotein phosphatase is required for asexual development and pathogenesis of Sclerotinia sclerotiorum. Mol. Plant-Microbe Interact. 20:944-954.

Flegg, C. P., Sharma, M., Medina-Palazon, C., Jamieson, C., Galea, M., Brocardo, M. G., Mills, K. and Henderson, B. R. 2010. Nuclear export and centrosome targeting of the protein phosphatase 2A subunit B56alpha: Role of B56alpha in nuclear export of the catalytic subunit. J. Biol. Chem. 285: 18144-18154.

Gallego, M. and Virshup, D. M. 2005. Protein serine/threonine phosphatases: life, death, and sleeping. Curr. Opin. Cell. Biol. 17:197-202. 
Gardiner, D. M., Kazan, K. and Manners, J. M. 2009. Novel genes of Fusarium graminearum that negatively regulate deoxynivalenol production and virulence. Mol. Plant-Microbe Interact. 22:1588-1600.

Hahn, K., Miranda, M., Francis, V. A., Vendrell, J., Zorzano, A. and Teleman, A. A. 2010. PP2A regulatory subunit PP2A-B' counteracts S6K phosphorylation. Cell Metab. 11:438-444.

Han, Y. K., Kim, M. D., Lee, S. H., Yun, S. H. and Lee, Y. W. 2007. A novel F-box protein involved in sexual development and pathogenesis in Gibberella zeae. Mol. Microbiol. 63:768779.

Kim, H. K., Lee, T. and Yun, S. H. 2008. A putative pheromone signaling pathway is dispensable for self-fertility in the homothallic ascomycete Gibberella zeae. Fungal Genet. Biol. 45:1188-1196.

Kim, H. K., Lee, Y. W. and Yun, S. H. 2011. GzRUM1, encoding an ortholog of human retinoblastoma binding protein 2, is required for ascospore development in Gibberella zeae. Plant Pathol. J. 27:20-25.

Kim, H. K. and Yun, S. H. 2011. Evaluation of potential reference genes for quantitative RT-PCR analysis in Fusarium graminearum under different culture conditions. Plant Pathol. J. 27:301-309.

Kosmidou, E., Lunness, P. and Doonan, J. H. 2001. A type 2A protein phosphatase gene from Aspergillus nidulans is involved in hyphal morphogenesis. Curr. Genet. 39:25-34.

Lechward, K., Awotunde, O. S., Swiatek, W. and Muszynska, G. 2001. Protein phosphatase 2A: variety of forms and diversity of functions. Acta Biochim. Pol. 48:921-933.

Lee, S. H., Han, Y. K., Yun, S. H. and Lee, Y. W. 2009. Roles of the glyoxylate and methylcitrate cycles in sexual development and virulence in the cereal pathogen Gibberella zeae. Eukaryot. Cell 8:1155-1164.

Leslie, J. F. and Summerell, B. A. 2006. The Fusarium lab manual. Blackwell, Ames, IA, U.S.A.

Liu, M., Parker, L. L., Wadzinski, B. E. and Shieh, B. H. 2000. Reversible phosphorylation of the signal transduction complex in Drosophila photoreceptors. J. Biol. Chem. 275: 12194-12199.

Louis, J. V., Martens, E., Borghgraef, P., Lambrecht, C., Sents, W., Longin, S., Zwaenepoel, K., Pijnenborg, R., Landrieu, I., Lippens, G., Ledermann, B., Gotz, J., Van Leuven, F., Goris, J. and Janssens, V. 2011. Mice lacking phosphatase PP2A subunit PR61/B'delta (PPP2R5D) develop spatially restricted tauopathy by deregulation of CDK5 and GSK3beta. Proc. Natl. Acad. Sci. USA 108:6957-6962.

Luan, S. 2003. Protein phosphatases in plants. Ann. Rev. Plant Biol. 54:63-92.

McCright, B., Rivers, A. M., Audlin, S. and Virshup, D. M. 1996. The b56 family of protein phosphatase 2A (PP2A) regulatory subunits encodes differentiation-induced phosphoproteins that target PP2A to both nucleus and cytoplasm. J. Biol. Chem. 271:22081-22089.

McMullen, M., Jones, R. and Gallenberg, D. 1997. Scab of wheat and barley: A re-emerging disease of devastating impact. Plant Dis. 81:1340-1348.

Mumby, M. 2007. PP2A: Unveiling a reluctant tumor suppressor. Cell 130:21-24.

Muneer, S., Ramalingam, V., Wyatt, R., Schultz, R. A., Minna, J. D. and Kamibayashi, C. 2002. Genomic organization and mapping of the gene encoding the PP2A B56gamma regulatory subunit. Genomics 79:344-348.

Namiki, F., Matsunaga, M., Okuda, M., Inoue, I., Nishi, K., Fujita, Y. and Tsuge, T. 2001. Mutation of an arginine biosynthesis gene causes reduced pathogenicity in Fusarium oxysporum $\mathrm{f}$. sp. melonis. Mol. Plant-Microbe Interact. 14:580-584.

O'Donnell, K., Kistler, H. C., Tacke, B. K. and Casper, H. H. 2000. Gene genealogies reveal global phylogeographic structure and reproductive isolation among lineages of Fusarium graminearum, the fungus causing wheat scab. Proc. Natl. Acad. Sci. USA 97:7905-7910.

Sambrook, J. and Russell, D. W. 2001. Molecular cloning: A laboratory manual. Cold Spring Harbor Laboratory Press, Plainview, U.S.A.

Semighini, C. P., Murray, N. and Harris, S. D. 2008. Inhibition of Fusarium graminearum growth and development by farnesol. FEMS Microbiol. Lett. 279:259-264.

Seo, B. K., Kim, H. K., Lee, Y. W. and Yun, S. H. 2007. Functional analysis of a histidine auxotrophic mutation in Gibberella zeae. Plant Pathol. J. 23:51-56.

Sontag, E. 2001. Protein phosphatase 2A: The trojan horse of cellular signaling. Cell Signal. 13:7-16.

Springer, M. L. and Yanofsky, C. 1989. A morphological and genetic analysis of conidiophore development in Neurospora crassa. Genes Dev. 3:559-571.

Yang, Y., He, Q., Cheng, P., Wrage, P., Yarden, O. and Liu, Y. 2004. Distinct roles for PP1 and PP2A in the Neurospora circadian clock. Genes Dev. 18:255-260.

Yatzkan, E., Szoor, B., Feher, Z., Dombradi, V. and Yarden, O. 1998. Protein phosphatase 2A is involved in hyphal growth of Neurospora crassa. Mol. Gen. Genet. 259:523-531.

Yatzkan, E. and Yarden, O. 1999. The b regulatory subunit of protein phosphatase $2 \mathrm{~A}$ is required for completion of macroconidiation and other developmental processes in Neurospora crassa. Mol. Microbiol. 31:197-209.

Yu, H. Y., Seo, J. A., Kim, J. E., Han, K. H., Shim, W. B., Yun, S. H. and Lee, Y. W. 2008. Functional analyses of heterotrimeric $\mathrm{G}$ protein $\mathrm{G}$ alpha and $\mathrm{G}$ beta subunits in Gibberella zeae. Microbiology 154:392-401.

Yu, J. H., Hamari, Z., Han, K. H., Seo, J. A., Reyes-Dominguez, Y. and Scazzocchio, C. 2004. Double-joint PCR: A pcr-based molecular tool for gene manipulations in filamentous fungi. Fungal Genet. Biol. 41:973-981.

Yun, S. H. 1998. Molecular genetics and manipulation of pathogenicity and mating determinants in Mycosphaerella zeaemaydis and Cochliobolus heterostrophus. Ph.D. dissertation, Cornell University, Ithaca, NY, U.S.A. 